\title{
Mott-Hubbard crossover in two-dimensional clusters threaded by a magnetic field
}

\author{
A.K. Jermakian ${ }^{\text {a,* }}$, A.N. Kocharian ${ }^{\text {, }}$ A.S. Saakian ${ }^{\mathrm{c}}$ \\ ${ }^{a}$ Department of Physics, New Jersey Institute of Technology, Newark, NJ 07012, USA \\ ${ }^{\mathrm{b}}$ Department of Physics, California State University, Northridge, CA 91330-8268, USA \\ ${ }^{\mathrm{c}}$ Armenian Engineering University, Terian St. 105, Yerevan 375009, Armenia
}

\begin{abstract}
The ground-state characteristics of mesoscopic Hubbard clusters in the high-spin region are investigated as a function of cluster dimensions, lattice geometry and electron interaction in response to an applied transverse magnetic field normal to the surface. It is shown that the critical magnetic field of the fully polarized state (in the presence of longitudinal magnetic field), which depends on the transverse field (Peierls phase), the interaction strength, the lattice geometry or the cluster size, can serve as a sensitive measure for the Mott-Hubbard crossover in $1 \mathrm{~d}$ and $2 \mathrm{~d}$ finite-size systems. An exact analytical calculation of the critical magnetic field for the infinite chain with next nearest-neighbor hopping is also performed and the condition for metal-insulator transition is derived. (C) 2000 Published by Elsevier Science B.V. All rights reserved.
\end{abstract}

Keywords: Mott-Hubbard crossover; Atomic clusters; Peierls phase

Despite considerable efforts the understanding of electron correlations and localization in low-dimensional systems and finite-size clusters is still far from complete [1-5]. Up to the present rigorous results for the Hubbard model are rare except in the one-dimensional case [6]. Evidently, the strong magnetic field results in ferromagnetic ordering and, using easily constructed elementary two-particle excitations, the problem at half-filling becomes exactly solvable in small atomic systems as well as for a discrete lattice in the thermodynamic limit [7-9]. The criteria for metal-insulator transition in the finitesize $1 \mathrm{~d}$ and $2 \mathrm{~d}$ Hubbard model with periodic boundary conditions was investigated in the presence of a strong longitudinal Zeeman magnetic field in Ref. [10]. The formation of bound electron-hole pairs (excitons) in the high-spin region gives rise to the crossover from the itinerant regime to the localized state, which depends on the lattice geometry, system size (and parity) and threaded magnetic flux (Peierls phase) [11]. Here we

\footnotetext{
* Corresponding author. Tel.: + 1-973-596-3557; fax: + 1973-596-5794.

E-mail address: jermakian@tesla.njit.edu (A.K. Jermakian)
}

present these results for two-dimensional coupled Hubbard chains in a strong magnetic field.

$$
\begin{aligned}
H= & \sum_{i>j, i^{\prime}>j^{\prime}, \sigma}\left\{\left[\exp (\mathrm{i} \alpha)\left(t c_{i \sigma}^{+} c_{j \sigma}+t_{1} c_{i^{\prime} \sigma}^{+} c_{j^{\prime} \sigma}\right)\right]+\text { h.c. }\right\} \\
& +\frac{U}{2} \sum_{i, \sigma} n_{i, \sigma} n_{i,-\sigma}-\frac{h}{2} \sum_{i, \sigma} n_{i, \sigma} \sigma .
\end{aligned}
$$

In addition to the usual nearest-neighbor hopping term $t$, we included the matrix element, $t_{1}$, for electron hopping between the next-nearest sites, both having the same phase Peierls phase $\alpha$. Previously obtained exact analytical results for $\mu \equiv U / 4 t$ as a function of $\eta \equiv h_{\mathrm{c}} / 4 t$ in $1 \mathrm{~d}$ and $2 \mathrm{~d}$ in the presence of a Peierls phase $\alpha$ allows us to analyze exact expressions for the critical magnetic field $h_{\mathrm{c}}$ [10] and its second derivative with respect to $\alpha$, $\mathrm{d}^{2} \eta / \mathrm{d} \alpha^{2}$, which is equivalent to the charge stiffness coefficient $\left.\mathrm{D}_{\mathrm{c}}[4,5]\right)$. First we examine the behavior of $\zeta$ and $\mathrm{d}^{2} \eta / \mathrm{d} \alpha^{2}$ as a function of $\mathrm{N} \alpha$ for a fixed value of $\mu=0.27$.

We found that the stable mode corresponds to $\bar{C}\left(N_{x} \theta\right)$ $\mp \cos \left(N_{x} \alpha\right)>0^{1}\left(\mp \Rightarrow\right.$ even, odd $\left.N_{\mathrm{x}}\right)$, which agrees with

\footnotetext{
${ }^{1} \bar{C}(N \theta)=\cosh (\theta) . \quad \zeta=\cos (\theta) \geqslant 1, \# \quad \bar{C}(N \theta)=\cos (\theta) . \quad \zeta=$ $\cosh (\theta)<1$.
} 


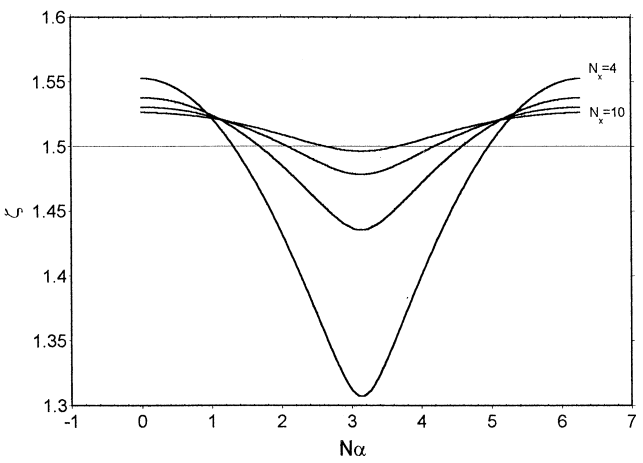

Fig. 1. Energy parameter $\zeta$ versus $N \alpha$ for $N_{x}=4,6,8,10$ and $N_{y}=3$.

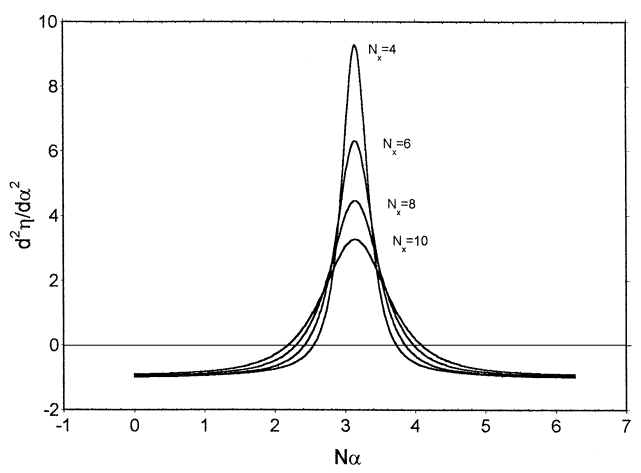

Fig. 2. Charge stiffness coeficient $\mathrm{D}_{\mathrm{c}}$ versus $N \alpha$ for $N_{x}=4,6,8,10$ and $N_{y}=3$.

the result [11] in the absence of Peierls phase $(\alpha=0)$. For a negative value we generate other modes which are less stable (lower value of $\zeta$ and $\eta$ ) which we do not consider here. Earlier we found that in $1 \mathrm{~d}$ case there is a transition from the localized regime to the itinerant regime when $\zeta=1[10,11]$. In the $2 d$ case, i.e. for coupled chains, there is also a crossover between localized and delocalized regimes. For coupled chains the crossover takes place at $\zeta=1+\cos \left(\pi / N_{y}\right)$ when $N_{y}$ is odd and $N_{x}$ is even or odd, and at $\zeta=2$, when both $N_{x}$ and $N_{y}$ are even. In Figs. 1 and 2 a horizontal line corresponding to this transition is also shown. Note that for $\zeta$ greater than the critical value $\zeta_{\text {cr }}$ at which this crossover occurs, results in electron-hole binding and localization, while values less than $\zeta_{\text {cr }}$ results in delocalization. The range of $N \alpha$ corresponding to the localized or delocalized region is also evident in these graphs. The character of variation the curves $\zeta$ versus $N \alpha$ depends only on the parity of $N_{x}$. Note that $2 \mathrm{~d}$ symmetry in the presence of Peierls phase is broken along the $x$ direction. The system with even parity is in a localized state for $\alpha=0$ and arbitrary $N_{x}$ and $N_{y}$, while a system with mixed parity is also localized only at relatively large $N_{x}$ limit. This has been explicitly shown for any finite value of $N_{x}$ and $N_{y}=1,2$, or 3 as well as for finite square lattices of $N_{x}=N_{y}$.

Having obtained the exact expression for $\mathrm{d}^{2} \eta / \mathrm{d} \alpha^{2}$, we examine the response of even and odd chains to the transverse field. When this parameter changes its sign it shows a critical value for the phase (transverse field) at which there occurs a crossover from localized to unbound state.

It is readily apparent that there is qualitative agreement between the two methods of determining the crossover, the range for behavior is a decreasing function of $N_{x}$, however the range of $N \alpha$ for the delocalized region is always smaller using the criterion $\mathrm{d}^{2} \eta / \mathrm{d} \alpha^{2}>0$, than when using the criterion $\zeta>\zeta_{\mathrm{cr}}$.

In conclusion our results for coupled Hubbard finite chains in two dimensions show that the ground-state response to the transverse field in the presence of a strong field near the magnetic saturation resembles the onedimensional case and depends on the number of even or odd $N_{x}$ lattice sites (parity) along the direction of the vector potential. The applied transverse field or the variation of the cluster size $\left(N_{x}\right)$ in finite $2 d$ lattices at halffilling results in the smooth Mott-Hubbard crossover from metallic (itinerant) to the insulating state. The variation of $\mathrm{d}^{2} \eta / \mathrm{d} \alpha^{2}$ with $\alpha$ serves as a measure for the observation of the Mott-Hubbard crossover in finite-size systems. Our results at large $N_{x}$ limit show the stability of an insulating state at infinitesimal $U$, consistent with $[6,7]$. In addition we performed analytical calculations $h_{\mathrm{cr}}$ versus $U$ for the nonbipartite infinite chain with the next nearest-neighbor hopping term $t_{1}$ and found the critical parameters for the Mott-Hubbard transition in the $1 \mathrm{~d}$ case.

\section{References}

[1] E. Dagotto, Rev. Mod. Phys. 66 (1994) 763.

[2] N. Yu, M. Fowler, Phys. Rev. B 45 (1992) 11795.

[3] R.M. Noack, S.R. White, D.J. Scalapino, Phys. Rev. Lett. 73 (1994) 882.

[4] B.S. Shastry, B. Sutherland, Phys. Rev. Lett. 65 (1990) 243.

[5] C.A. Stafford, A.J. Millis, B.S. Shastry, Phys. Rev. B 43 (1991) 13660.

[6] H. Frahm, V. Korepin, Phys. Rev. B 42 (1990) 10553.

[7] Q.P. Li, R. Joint, Phys. Rev. B 47 (1993) 3979.

[8] Q.P. Li, R. Joint, Phys. Rev. B 49 (1994) 1632.

[9] A.N. Kocharian, J. Sebold, Phys. Rev. B 53 (1996) 12804.

[10] A.N. Kocharian, A. Jermakian, A. Sogomonian, Physica B 230-232 (1997) 1031.

[11] A.N. Kocharian, A. Jermakian, A. Saakian, Physica B 239-261 (1999) 736. 